\title{
MULTI-SCALE MODELLING OF SNOW MICROSTRUCTURE
}

\author{
A. Carbone, ${ }^{1,3, *}$ B. M. Chiaia, ${ }^{2}$ B. Frigo, ${ }^{2}$ \& C. Türk ${ }^{1}$ \\ ${ }^{1}$ Applied Science and Technology Department, Politecnico di Torino, Corso Duca degli Abruzzi \\ 24, I-10129 Torino, Italy \\ ${ }^{2}$ Department of Structural and Geotechnical Engineering, Politecnico di Torino, Corso Duca \\ degli Abruzzi 24, I-10129 Torino, Italy \\ ${ }^{3}$ ETH Zurich, Switzerland
}

*Address all correspondence to A. Carbone, E-mail: anna.carbone@polito.it

\begin{abstract}
A three-dimensional multiscale spatial model of snow with evolving microstructure is presented. Many engineering and environmental problems require a comprehensive understanding of snow behavior which arises as a consequence of phenomena spanning a wide spectrum of spatial length scales. Snow is classically described as a granular heterogeneous medium consisting of air and three water phases: ice, vapor, and liquid. The ice phase consists of grains arranged on a matrix according to a random load-bearing skeleton. The challenge is to achieve a detailed description of the mechanical and morphological characteristics of different snow microstructures that may have the same global density. Snow density can be determined by in situ measurements with quite good accuracy, and by means of the box-counting method, the fractal dimension of snow samples characterized by grains with different diameters could be determined. It was suggested that the fractal dimension can be adopted as a relevant parameter for quantifying snow morphology, in terms of the distribution of voids, and density over a wide range of spatial scales. In this work this concept is further developed. Snow density is simulated by means of a lacunar fractal, namely, a generalized Menger sponge. Then, a fully threedimensional invasive stochastic fractal model is adopted. This model performs a three-dimensional mapping of the snow density to a three-dimensional fractional Brownian field. In particular, snow samples with evolving microstructure are quantified as a continuous function of the fractal dimension.
\end{abstract}

KEY WORDS: snow physics, porous media, three-dimensional fractal models, Hurst exponent

\section{INTRODUCTION}

Understanding the fundamental phenomena by which snow microstructure evolves is becoming increasingly relevant for scientific communities interested in environment, geophysics, and engineering. Snow is a porous material with a solid ice crystal skeleton (grains) with air and water vapor mixture embedded in the pores (Blackford, 2007; Colbeck et al., 1985). However, the classification of snow as a single material is difficult. Snow is a multifaceted material exhibiting a multitude of forms. Due to its close existence to its melting point, snow microstructure changes with time. The type, size, and shape of snow grains and inter-grain bonds change accordingly and affect the cohesive strength of the snow cover. Snow metamorphism strongly depends on the temperature gradient within the snow, according to various energy fluxes through the snow cover, for example, visible and infrared radiation, geothermic heat. Ice sublimates in the snow cover, flows along existing vapor gradients, and condensates at colder positions of the ice matrix. Physical and mechanical properties of snow greatly vary over space and rapidly evolve in time, affecting density and texture. The former is defined as the mass per unit volume, while the latter defines the shape, size, and bonds of the grains constituting the ice skeleton. Density and texture depend on meteorological conditions (essentially temperature, but also humidity) which, together with the mechanical strains, cause metamorphism and, ultimately, instability of snow cover. Both features strongly influence the natural porosity of snow and its thermal, chemical, and 
mechanical properties (Dadic et al., 2008; Fauve et al., 2008; Golubev and Frolov, 1998; Frolov and Fedyukin, 1998; Kaempfer et al., 2005; Kaempfer et al., 2007; Kaempfer and Schneebeli, 2007; Kaempfer and Plapp, 2009). Density is the parameter usually adopted to classify and assess snow properties, thanks to the simplicity of in situ measurements. In general, the density is determined by excavating snow trenches and weighing given volumes of snow (Colbeck et al., 1985). This procedure enables the density to be adopted as a parameter for quantifying snow characteristics such as viscosity, shear stress and strength, cohesion, and mechanical properties, e.g., Young's modulus, Poisson's ratio. However, completely different mechanical features might correspond to different types of snow having the same density (Shapiro et al., 1997). Physical properties of snow are commonly described in terms of specific density, which is defined as the ratio between snow density $\rho_{\text {snow }}$ and ice density $\rho_{\text {ice. }}$. In particular, Kirchner et al. (2001) and Petrovic (2003) work out power-law relationships with noninteger exponents between mechanical properties (i.e., tension and shear strength, toughness, etc.) and specific density, suggesting fractal snow features.

Multiscale modelling and fractional calculus have been extensively adopted to characterize different classes of materials (Ostoja-Starzewski 1998, 1999, 2006; Turcotte, 1997). In particular, snow crystals and snowflake shapes have already been modelled by using the von Koch snowflake curves and iterated function systems. In the last two decades, fractal snow cover has resulted in several applications: from the measure of fractal dimension of images, to remote sensing and mapping of snow cover and depth distribution by satellite and Lidar images, from the study of the roughness of the snowpack, to the determination of the air flux across snow surfaces, and the definition of the spatial variation of the snow water equivalent (Deems et al., 2006, 2008; Emerson et al., 1999; Fassnacht et al., 2006; Fassnacht et al., 2009; Shook et al., 1993). By analyzing three-dimensional tomographies obtained for cubic samples with different densities, the granular structure and the spatial distribution of voids have been recently investigated at the Centre Etudes de Neige of Météo France (www.cnrm.meteo.fr/passion/neige1.htm) and their fractal character clearly detected (Faillettaz, 2003). Chiaia and Frigo (2009) have described the probability of occurrence of snow avalanche events by assuming scale invariance of the snowpack at the smallest scales with the consequent scale invariance behavior at the largest scales, allowing the stability of the snow cover at larger scales.

By adopting a fully three-dimensional fractal description and analysis of the distribution of ice grains in a snow cube (Carbone, 2007; Arianos and Carbone, 2009; Türk et al., 2010), the multiscale character of snow is quantified in deeper detail. Considering the stochastic peculiarities of the grain distribution of snow samples, a fully threedimensional stochastic fractal model is presented, able to reproduce the local randomness of a real snow sample microstructure. This fully three-dimensional stochastic fractal model is used to relate snow texture and density as a function of Hurst exponent. The proposed model provides a fully three-dimensional analysis in terms of a continuum fractional Brownian field as opposed to the discrete Menger model description. Within the framework of this model, fractal dimension $D$ and Hurst exponent $H$ optimally quantify ice distribution and accurately reproduce the values of snow density for different microstructures.

The paper is organized as follows. In Section 2, snow density is simulated by means of the generalized Menger sponge model, which is characterized by a discrete set of fractal dimension. In Section 3, the fully three-dimensional stochastic fractional Brownian model is reported. This model has the advantage of reproducing the randomness of the local microstructure of snow samples in a more realistic way. In particular, snow density has been mapped to a three-dimensional Brownian field, with the Hurst exponent $H$ continuously ranging from 0 to 1 .

\section{GENERALIZED MENGER SPONGE MODEL}

The Menger sponge is a lacunar fractal generated according to the following procedure. First, a bulky cube is divided into $3 \times 3 \times 3=27$ equal subcubes. Then, seven subcubes are removed from the center of each face and from the center of the cube, resulting in $N_{f}=20$ filled subcubes and $N_{e}=3^{3}-N_{f}=7$ empty subcubes. This single step will be repeatedly applied to the remaining cubes. For a solid cube with linear size $r_{0}$, the subcubes of the 1st-level Menger sponge are characterized by linear size $r_{1}=r_{0} / 3$. The 2nd-level Menger sponge is characterized by subcubes with $r_{2}=r_{0} / 9$ [Fig. 1(a)]. $r_{i}=r_{0} / 3^{i}$ is the linear dimension of the subcubes at the iteration $i$.

The Menger sponge has been used to model porous media, whose relevant parameter is the void index or porosity $\phi$ (Turcotte 1997). For the Menger sponge, the porosity $\phi_{i}$, defined as the relative volume of voids per unit volume, can be expressed by the following relationship: 


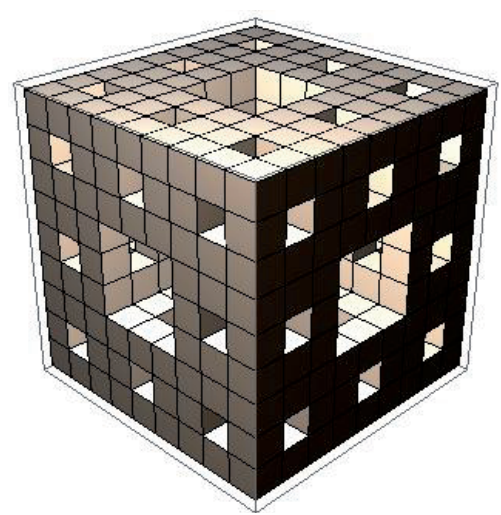

(a)

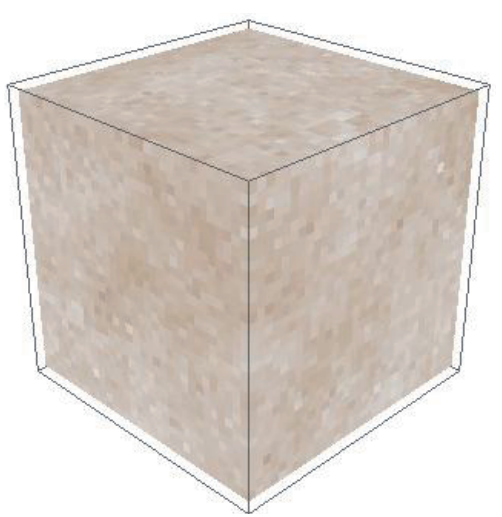

(b)

FIG. 1: (a) Menger sponge at the second iteration where the size, density, and porosity are, respectively, $r_{2}=9 r_{0}$, $\rho_{2}=(400 / 729) \rho_{0}$, and $\phi_{2}=329 / 729$. (b) Three-dimensional invasive fractal generated with the Hurst exponent $H=0.1$ according to the three-dimensional fractional Brownian model. The density $\rho(r)$ ranges between 0 and 917 $\mathrm{kg} / \mathrm{m}^{3}$, with a size ratio of $400 \times 400 \times 400$ values.

$$
\phi_{i}=1-\left(\frac{\rho_{i}}{\rho_{0}}\right),
$$

where $\rho_{0}$ is the density of the initial cube, and $\rho_{i}$ is the density of the Menger sponge obtained at the iteration $i$. By taking into account that $\rho_{i}$ and $\rho_{0}$ are inversely proportional to the volumes and, then, to the linear sizes $r_{0}$ and $r_{i}$, Eq. (1) can be written as

$$
\phi_{i}=1-\left(\frac{r_{0}}{r_{i}}\right)^{3-D}
$$

where $D$ is the fractal dimension, that is the scale-independent parameter characterizing the morphology of a porous material. The fractal dimension of the Menger sponge is given by $D=\ln N_{f} / \ln 3$. Here we assume self-similarity which implies a linear relationship $H=3-D$ between fractal dimension $D$ and Hurst coefficient $H$ (Gneiting and Schlather, 2004; Mateu et al., 2007). Therefore, the density and void index of the $i$ th-level Menger sponge, as a function of the linear dimension of cube $r_{0}$ and subcubes $r_{i}$ can be written as

$$
\frac{\rho_{i}}{\rho_{0}}=\left(\frac{r_{0}}{r_{i}}\right)^{3-D}=\left(\frac{r_{0}}{r_{i}}\right)^{3-\ln N_{f} / \ln 3}=\left(\frac{r_{0}}{r_{i}}\right)^{H} .
$$

For example, for the Menger sponge at the second iteration shown in Fig. 1(a), the porosity is $\phi_{2}=329 / 729$ and the density is $\rho_{2}=400 \rho_{0} / 729$. The procedure described above for generating the Menger sponge can be generalized by removing an arbitrary number $N_{e}$ of subcubes (instead of seven) out of an arbitrary number of filled cubes $N_{f}$ (instead of 20). This generalized construction results in fractal structures with a Hurst exponent different than $H=0.273$.

To model snow samples, a solid ice cube characterized by density $\rho_{\text {ice }}=\rho_{0}=917 \mathrm{~kg} / \mathrm{m}^{3}$ and linear size $r_{\text {ice grain }}=r_{0}$ is considered as the initial cube. Then snow is simulated as a Menger fractal form of ice characterized by density $\rho_{\text {snow }}=\rho_{i}$ and linear size $r_{\text {snow }}=r_{i}$ at the $i$ th level. Thus Eq. (3) is rewritten as follows:

$$
\frac{\rho_{\text {snow }}}{\rho_{\text {ice }}}=\left(\frac{r_{\text {ice grain }}}{r_{\text {snow }}}\right)^{3-\ln N_{f} / \ln 3}=\left(\frac{r_{\text {ice grain }}}{r_{\text {snow }}}\right)^{3-D}=\left(\frac{r_{\text {ice grain }}}{r_{\text {snow }}}\right)^{H} .
$$

Fractal dimension $D$ and Hurst exponent $H$ can be used to characterize the microstructure of the medium, enabling a measure of ice porosity of the snow sample. The fractal character of snow has recently been studied using images obtained on various snow cubic samples with different densities. These images show the granular structure of snow and 
the spatial distribution of its voids. By means of the box-counting method, the fractal dimension of four snow samples, characterized by grains with different diameter, could be determined (Faillettaz, 2003). By applying the generalized Menger sponge model and using the same size of the samples: $r_{\text {ice grain }}=0.25 \mathrm{~mm}$ and $r_{\text {snow }}=r_{0}=100 \mathrm{~mm}$, the fractal dimension $D$ of different classes of snow can be calculated (Table 1). By analyzing the density and void index as a function of the linear sample dimension $r_{i}$, we observe that as the sample size increases, snow differs more and more from ice. At small scales, ice and snow approximately show the same behavior, while the spatial variability of the density does not greatly influence the mechanical properties. Therefore we argue that snow density is a function of the scale and the probability to find large defects (e.g., superweak zones in a weak layer) increases with the dimension of the snow cover, as, for example, providing more intrinsic brittleness for large snow slopes (Chiaia and Frigo, 2009; Chiaia et al., 2008). Numerical results reported in Table 1 confirm that $D$ is an accurate measure of the distribution of the ice mass into snow samples. We also observe that the values of the fractal dimension measured by the boxcounting method (Faillettaz, 2003) are consistent with the values calculated by the generalized Menger sponge model (Table 2).

\section{RANDOM FRACTAL MODEL}

Stochastic fractals, generated through the randomization of deterministic fractals for example, might have the advantage to reproduce the microstructure of disordered materials with higher accuracy and richer details. In the previous section the generalized Menger sponge model and its fractal dimension concept, characterized by scale invariant porosity, is used to describe the density of snow samples with different microstructures. However, even if this model is of significant practical relevance, it does not fully capture the randomness of the local fractal structure of snow samples. It is due to the discrete set of fractal dimension values. Moreover, the porosity of a real-world fractal should be free of scale, requiring an infinite number of iterations of the process generating the sponge, which is practically impossible to achieve. On account of these limitations and keeping in mind that snow can be described as a sin-

TABLE 1: Fractal dimension $D$ and Hurst exponent $H$ for snow samples with $r_{\text {ice grain }}=0.25 \mathrm{~mm}$ and $r_{\text {snow }}=100 \mathrm{~mm}$ calculated according to the generalized Menger sponge model.

\begin{tabular}{cccc}
\hline \hline Snow type & Density $\left(\mathbf{k g} / \mathbf{m}^{3}\right)$ & Fractal dimension & Hurst exponent \\
\hline \hline Dry snow & $50<\rho<200$ & $2.5145<D<2.7458$ & $0.4855<H<0.2542$ \\
Snow & $200<\rho<550$ & $2.7458<D<2.9147$ & $0.2542<H<0.0853$ \\
Firn & $550<\rho<820$ & $2.9147<D<2.9813$ & $0.2542<H<0.0853$ \\
Porous ice & $820<\rho<917$ & $2.9813<D<3.0000$ & $0.0186<H<0.0000$ \\
Ice & 917 & 3 & 0 \\
\hline \hline
\end{tabular}

TABLE 2: Fractal dimension and Hurst exponent for different snow samples. The values obtained by means of the box-counting method are in the 3rd and 4th column (Faillettaz, 2003). The values reported the 5th and 6th columns have been calculated by using the generalized Menger sponge model of Section 2.

\begin{tabular}{cccccc}
\hline \hline Snow samples & Density $\mathbf{( k g / \mathbf { m } ^ { 3 } )}$ & $D_{\text {Box }}$ & $H_{\text {Box }}$ & $D_{\text {Sponge }}$ & $H_{\text {Sponge }}$ \\
\hline \hline Fine grains & 200 & 2.62 & 0.38 & 2.805 & 0.195 \\
Fine grains (Huez) & 200 & 2.83 & 0.17 & 2.805 & 0.195 \\
Fine grains & 300 & 2.80 & 0.20 & 2.857 & 0.143 \\
Rounded grains & 300 & 3.00 & 0.00 & 2.857 & 0.143 \\
\hline \hline
\end{tabular}


tered porous material consisting of a continuous ice network and a continuous pore space (Heggli et al., 2009; Flin et al., 2003), in this work a three-dimensional generalization of the random midpoint displacement algorithm is implemented (Türk et al., 2010) to model snow as a three-dimensional fractal heterogeneous medium. The method for generating compact fractal disordered media is based on fractional Brownian functions, which are characterized by a correlation function depending, as a power law, on the distance between the points on a square or cubic grid. Here the random midpoint displacement algorithm is applied over a three-dimensional grid and a fractal cube is obtained. The procedure is based on the function which defines the values of the equally spaced three-dimensional lattice as

$$
f_{H}(r)=\frac{1}{2^{3}} \sum_{k} f_{k}(r)+\sigma_{j, 3}
$$

with $r=\left(i_{1}, i_{2}, i_{3}\right)$ and lattice size $N=\left(N_{1}, N_{2}, N_{3}\right)$. The sum is calculated over the $k$ end points of the lattice, and the quantity $\sigma_{j, 3}$ is a random variable defined at each iteration $j$ as

$$
\sigma_{j, 3}^{2}=\sigma_{0}^{2}\left(\frac{\sqrt{3} N}{2^{j}}\right)^{2 H}\left[1-2^{2(H-3)}\right]
$$

where $\sigma_{0}$ is drawn from a Gaussian distribution with zero mean and unit variance. The Hurst exponent $H$ is the input and ranges from 0 to 1 . Initially, the lattice is fully homogeneous, with the function describing the fractal property taken as a constant, e.g., $f_{H}(r)=0$. Then the algorithm is implemented at each iteration $j$ according to the following procedure: the values of the function $f_{H, j}(r)$ are seeded as random variables at the eight vertices defining the cube; the value assigned to the central point is obtained by means of Eqs. (5) and (6), by using the eight vertices as input; the values located at the center of each face are assigned in the same way, but with the sum over the corresponding four vertices; finally, the midpoint of each of the 12 edges are calculated with the sum taken over the vertices at the end points of the edges. The first iteration of this algorithm results in 27 subcubes, and these steps, except for the initial seeds of the eight vertices, are iteratively repeated for each of the 27 subcubes. Eventually, the number of subcubes will become $\left(3^{j}\right)^{d}$, where $j$ is the iteration number and $d=3$.

By employing the detrending moving average (DMA) algorithm (Carbone, 2007; Carbone and Stanley, 2007), the Hurst exponent of the three-dimensional fractal structure can be subsequently checked. The core of the DMA algorithm is based on the generalized variance $\sigma_{D M A}^{2}(s)$, that for $d=3$ is written

$$
\sigma_{D M A}^{2}(s)=\frac{1}{V} \sum_{V}\left[f_{H}(r)-\tilde{f}_{n_{1}, n_{2}, n_{3}}(r)\right]^{2},
$$

where $f_{H}(r)=f_{H}\left(i_{1}, i_{2}, i_{3}\right)$ is the fractional Brownian field with $i_{1}=1,2, \ldots, N_{1}, i_{2}=1,2, \ldots, N_{2}$, and $i_{3}=$ $1,2, \ldots, N_{3}$. The function $\tilde{f}_{n_{1}, n_{2}, n_{3}}(r)$ is given by

$$
\tilde{f}_{n_{1}, n_{2}, n_{3}}(r)=\frac{1}{v} \sum_{k_{1}} \sum_{k_{2}} \sum_{k_{3}} f_{H}\left(i_{1}-k_{1}, i_{2}-k_{2}, i_{3}-k_{3}\right),
$$

with the size of the subcubes $\left(n_{1}, n_{2}, n_{3}\right)$ ranging from $(3,3,3)$ to the maximum values $\left(n_{1 \max }, n_{2 \max }, n_{3 \max }\right)$. $v=n_{1} n_{2} n_{3}$ is the volume of the subcubes. The quantity $V=\left(N_{1}-n_{1 \max }\right)\left(N_{2}-n_{2 \max }\right)\left(N_{3}-n_{3 \max }\right)$ is the volume of the fractal cube over which the average $\tilde{f}$ is defined. Equations (7) and (8) are defined for any geometry of the subarrays. However, in practice it is computationally more suitable to work with $n_{1}=n_{2}=n_{3}$ to avoid spurious directionality and biases in the calculations. The generalized variance $\sigma_{D M A}^{2}(s)$ scales as $\left(n_{1}^{2}+n_{2}^{2}+n_{3}^{2}\right)^{H}$ because of the fundamental property of fraction Brownian functions. In Fig. 2, the log-log plots of $\sigma_{D M A}^{2}(s)$ vs $s$ are shown for fractal cubes generated according to the procedure described above. The cubes have a Hurst exponent ranging from 0.1 to 0.9 with step 0.1 , and the plots of $\sigma_{D M A}^{2}(s)$ as a function $s$ are linear according to the expected power-law behavior

$$
\sigma_{D M A}^{2}(s) \propto\left(n_{1}^{2}+n_{2}^{2}+n_{3}^{2}\right)^{H} \propto s^{H}
$$

Volume 11, Number 2, 2013 


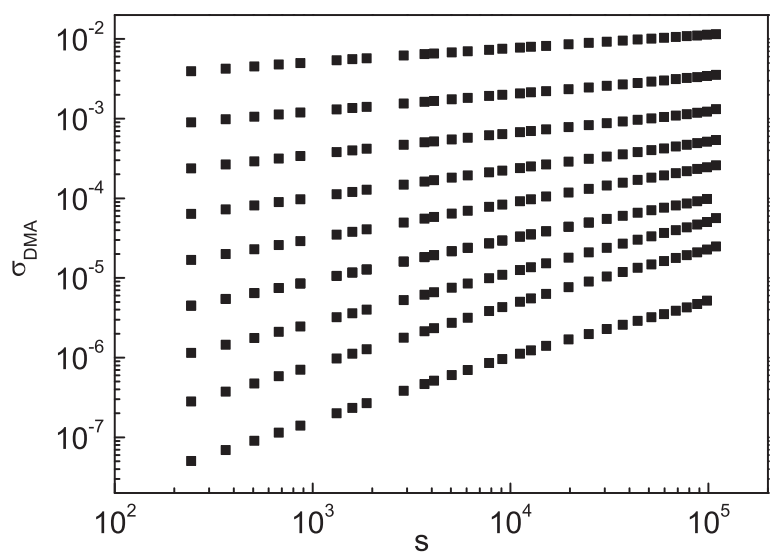

FIG. 2: $\log -\log$ plot of the function $\sigma_{D M A}$ as a function of scale $s$. The Hurst exponent is estimated by the slope of the curves, varying from 0.1 to 0.9 with step 0.1 .

Hence, the fractality of snow structure can be related to the density by mapping the fractional Brownian field $f_{H}(r)$ to a density field $\rho(r)$. Consequently, in this representation, the Hurst exponent, varying as a continuous parameter, should be intended as an index of specific snow compactness. Different snow textures are obtained by setting the Hurst exponent as a constant input parameter and varying the minimum value of the density $\rho_{\min }$ between 0 and $917 \mathrm{~kg} / \mathrm{m}^{3}$, while the maximum density is constant and equal to the ice density $\rho_{\max }=\rho_{\text {ice }}=917 \mathrm{~kg} / \mathrm{m}^{3}$.

In Fig. 1(b) snow structure corresponding to a cube with size $r_{0}=100 \mathrm{~mm}$ and granular size $r_{\text {ice grain }}=0.25 \mathrm{~mm}$ is shown generated by using the algorithm with $H=0.1$ and density range of $0-917 \mathrm{~kg} / \mathrm{m}^{3}$. One can observe how the ice structure is represented by an almost fully porous media featured by several areas of lower density. Colors are scaled in such a way that darker areas correspond to higher densities. The average densities $\rho_{\text {average }}$ have been calculated by taking $\rho_{\max }$ as the ice density $\rho_{0}=917 \mathrm{~kg} / \mathrm{m}^{3}$, while $\rho_{\min }$ is varied from 0 to $900 \mathrm{~kg} / \mathrm{m}^{3}$, as shown in Fig. 3. One can notice that the average density decreases more rapidly with lower values of $\rho_{\min }$ as the Hurst exponent decreases. In fact, both the structure and average density are practically unchanged as $H$ is changed, by

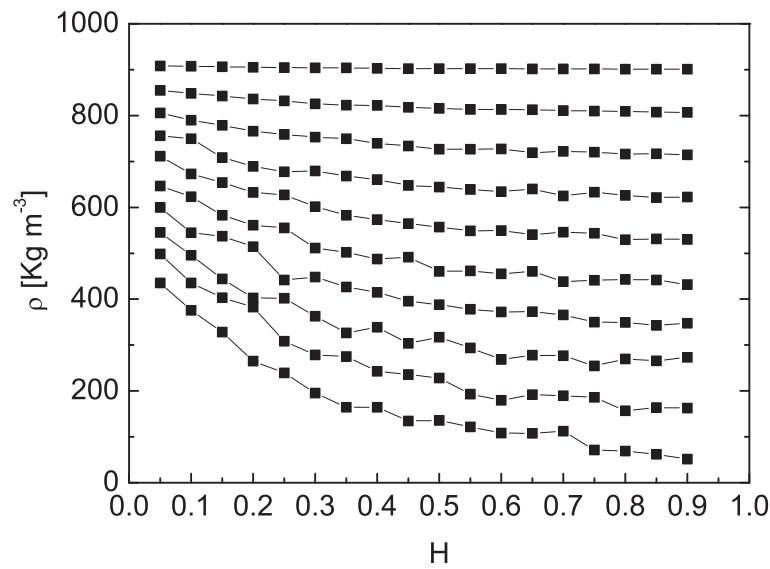

FIG. 3: Snow density as a function of the Hurst exponent. The different curves have been obtained by generating fractal cubes with different Hurst exponent. Then the fractal function has been mapped to a density function. In order to simulate different snow microstructures, the minimum value of the density $\rho_{\text {min }}$ has been varied between 0 and $917 \mathrm{~kg} / \mathrm{m}^{3}$, while the maximum value of the density is constant and equal to the ice density $\rho_{\text {max }}=\rho_{\text {ice }}=917 \mathrm{~kg} / \mathrm{m}^{3}$. Different curves, from bottom to top, correspond to values of $\rho_{\min }$ ranging from 0 and $917 \mathrm{~kg} / \mathrm{m}^{3}$ with step $100 \mathrm{~kg} / \mathrm{m}^{3}$. 
setting $\rho_{\min }=900 \mathrm{~kg} / \mathrm{m}^{3}$. On the other hand, by setting $\rho_{\min }=0 \mathrm{~kg} / \mathrm{m}^{3}$, the average density significantly changes as the Hurst exponent changes.

\section{CONCLUSIONS}

In order to capture the local heterogeneity of real snow samples, a three-dimensional random fractal model has been implemented with the ability to relate the snow microstructure variability to its density. Several physical and mechanical properties of snow are commonly expressed as a function of the density (or the related porosity and specific density) due to its simplicity of in situ measurements. Unfortunately, the density is not uniquely related to the microstructure of snow (different snow microstructure exhibits the same global density) and it varies with the scale. Thanks to this model, one should be able to investigate how the local structure changes according to the fractal dimension in relation to other physical properties. The present work is addressed to the development of the multiscale modelling of snow in a fully three-dimensional fractal framework (Carbone, 2007; Arianos and Carbone, 2009; Türk et al., 2010), relevant to the validation of experimental results, such as those reported by Gay et al. (2002) and Nakamura et al. (2001), and the description of the physical and mechanical properties which are of great importance for many applications.

The aim is the definition of a unique scale invariant parameter able to quantify the degree of snow metamorphism. The multiscale character of snow density and porosity has been analyzed by (i) a generalized Menger sponge and (ii) a stochastic fractional Brownian field. The former underlines that the Hurst exponent should be intended as a measure of the space-filling properties of snow, which can be related to the specific density. By comparison with data obtained from in situ measurements, the present approach shows that different Hurst exponents correspond to the same value of density, implying that density alone does not yield a complete information about snow microstructure.

\section{ACKNOWLEDGMENTS}

Fondazione CRT, - Progetto Alfieri, the Operational Programme "Italy - France (Alps - ALCOTRA)", Project "DynAval - Dynamique des Avalanches: Départ et interactions écoulement/obstacles," Regione Piemonte, Regione Autonoma Valle d'Aosta, and Politecnico di Torino are gratefully acknowledged for financial support. CINECA is gratefully acknowledged for CPU time at the high-performance computing (HPC) environment.

\section{REFERENCES}

Arianos, S. and Carbone, A., Cross-correlation of long-range correlated series, J. Stat. Mech: Theory Exp., p. $03037,2009$.

Blackford, J. R., Sintering and microstructure of ice: A review, J. Phys. D Appl. Phys., vol. 40, pp. R355-R385, 2007.

Carbone, A., Algorithm to estimate the Hurst exponent of high-dimensional fractals, Phys. Rev. E, vol. 76, p. $056703,2007$.

Carbone, A. and Stanley, H. E., Directed self-organized critical patterns emerging from fractional Brownian paths, Phys. A, vol. 340, pp. 544-551, 2004.

Carbone, A. and Stanley, H. E., Scaling properties and entropy of long range correlated series, Phys. A, vol. 384, pp. 21-24, 2007.

Carbone, A., Chiaia, B. M., Frigo, B., and Türk, C., Snow metamorphism: A fractal approach, Phys. Rev. E, vol. 81, p. 026706, 2010.

Chiaia, B. M., Cornetti, P., and Frigo, B., Triggering of dry snow slab avalanches: Stress versus fracture mechanical approach, Cold Regions Sci. Technol., vol. 53, pp. 170-178, 2008.

Chiaia, B. and Frigo, B., A scale-invariant model for snow avalanches, J. Stat. Mech.: Theory Exp., P02056, pp. 1-16, 2009.

Colbeck, S., Akitaya, E., Armstrong, R., Gubler, H., Lafeuille, J., Lied, K., McClung, D., and Morris, E., The International Classification for Seasonal Snow on the Ground, International Association of Scientific Hydrology, p. 37, 1985.

Dadic, R., Schneebeli, M., Lehning, M., Hutterli, M. A., and Ohmura, A., Impact of the microstructure of snow on its temperature: A model validation with measurements from Summit, Greenland, J. Geophys. Res., vol. 113, p. D14303, 2008.

Deems, J. S., Fassnacht, S. R., and Elder, K. J., Fractal distribution of snow depth from lidar data, J. Hydrometeor., vol. 7, pp. 285-297, 2006.

Deems, J. S., Fassnacht, S. R., and Elder, K. J., Interannual consistency in fractal snow depth patterns at two Colorado mountains, J. Hydrometeor, vol. 9, pp. 977-988, 2008.

Volume 11, Number 2, 2013 
Emerson, C. W., Lam, N. S., and Quattrochi, D. A., Multi-scale fractal analysis of image texture and pattern, Photogramm. Eng. Remote Sens., vol. 65, pp. 51-61, 1999.

Faillettaz, J., Le Déclenchement des Avalanches de Plaque de Neige: de L'approche Mécanique á L'approche Statistique, PhD, Université Joseph Fourier, Grenoble I, 2003.

Fassnacht, S. R. and Deems, J. S., Measurement sampling and scaling for deep montane snow depth data, Hydrolog. Process., vol. 20, pp. 829-838, 2006.

Fassnacht, S. R., Williams, M. W., and Corrao, M. V., Changes in the surface roughness of snow from millimetre to metre scales Ecol. Complexity, vol. 6, pp. 221-229, 2009.

Fauve, M., Hansueli, R., Lüthi, A., Schneebeli, M., and Lehning, M., Putting snow knowledge into the development of winter sports equipment, Sports Technol., vol. 1, pp. 145-151, 2008.

Flin, F., Brzoska, J. B., Lesaffre, B., Coléou, C., Pieritz, R. A., Fully three-dimensional modelling of curvature-dependent snow metamorphism, J. Phys. D: Appl. Phys., vol. 36, pp. A49-A54, 2003.

Frolov, A. D. and Fedyukin, I. V., Elastic properties of snow-ice formation in their whole, Annals Glaciology, vol. 26, pp. 55-58, 1998.

Gay, M., Fily, M., Genthon, C., Frezzotti, M., Oerter, H., and Winther, J. G., Snow grain-size measurements in Antarctica, J. Glaciology, vol. 48, no. 163, pp. 527-535, 2002.

Gneiting, T. and Schlather, M., Stochastic models that separate fractal dimension and the Hurst effect,SIAM Rev., vol. 46, pp. 269-282, 2004.

Golubev, V. N. and Frolov, A. D., Modelling the change in structure and mechanical properties in dry-snow densification to ice, Annals of Glaciology, vol. 26, pp. 45-50, 1998.

Heggli, M., Frei, E., and Schneebeli, M., Snow replica method for three-dimensional X-ray microtomographic imaging, J. Glaciology, vol. 55, no. 192, pp. 631-639, 2009.

Kaempfer, T. U., Schneebeli, M., and Sokratov, S. A., A microstructural approach to model heat transfer in snow, Geophys. Res. Lett., vol. 32, p. L21503, 2005.

Kaempfer, T. U., Hopkins, M. A., and Perovich, D. K., A three-dimensional microstructure-based photon-tracking model of radiative transfer in snow, J. Geophys. Res., vol. 112, p. D24113, 2007.

Kaempfer, T. U. and Schneebeli, M., Observation of isothermal metamorphism of new snow and interpretation as a sintering process, J. Geophys. Res., vol. 112, p. D24101, 2007.

Kaempfer, T. U. and Plapp, M., Phase-field modeling of dry snow metamorphism, Phys. Rev. E, vol. 79, p. $031502,2009$.

Mateu, J., Porcu, E., and Nicolis, O., A note on decoupling of local and global behaviours for the Dagum random field, Probab. Eng. Mech., vol. 22, no. 4, pp. 320-329, 2007.

Nakamura, T., Abe, O., Hasegawa, T., Tamura, R., and Ohta, T., Spectral reflectance of snow with a known grain-size distribution in successive metamorphism, Cold Regions Sci. Technol., vol. 32, pp. 13-26, 2001.

Kirchner, H. O. K., Michot, G., Narita, H., and Suzuki, T., Snow as a foam of ice: Plasticity, fracture and the brittle-to-ductile transition, Philos. Mag. A, vol. 81, no. 9, pp. 2161-2181, 2001.

Ostoja-Starzewski, M., Material spatial randomness: From statistical to representative volume elment, Probab. Eng. Mech., vol. 21, pp. 112-132, 2006.

Ostoja-Starzewski, M., Random field models of heterogeneous materials, Int. J. Solids Struct., vol. 21, pp. 2429-2455, 1998.

Ostoja-Starzewski, M., Scale effects in materials with random distributions of needles and cracks, Mech. Materials, vol. 31, no. 12, pp. 883-893, 1999.

Petrovic, J. J., Review of mechanical properties of ice and snow, J. Mater. Sci., vol. 38, pp. 1-6, 2003.

Shapiro, L. H., Johnson, J. B., Sturm, M., and Blaisdell, G. L., Snow mechanics review of the state of knowledge and applications, CRREL Report no. 97-3, 40, 1997.

Shook, K., Gray, D. M., and Pomeroy, J. W., The Geometry of PatchySnowcovers, 50th Eastern Snow Conf. and the 61st Western Snow Conf., Quebec City, Canada, pp. 89-96, 1993.

Turcotte, D. L., Fractal and Chaos in Geology and Geophysics, pp. 20-34, Cambridge University Press, Cambridge, UK, 1997.

Türk, C., Carbone, A., and Chiaia, B. M., Fractal heterogeneous media, Phys. Rev. E, vol. 81, p. 026706, 2010. 